\title{
Corrigendum
}

\section{Corrigendum to "Study on Inlet and Engine Integrated Model with Normal Shock Position Feedback"}

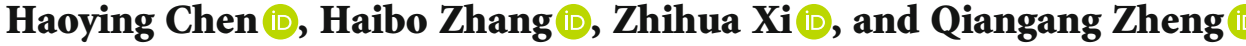 \\ Jiangsu Province Key Laboratory of Aerospace Power System, College of Energy and Power Engineering, Nanjing University of \\ Aeronautics and Astronautics, No. 29 Yudao Street, Nanjing 210016, China \\ Correspondence should be addressed to Haoying Chen; hychen_1029@nuaa.edu.cn \\ Received 18 November 2020; Accepted 18 November 2020; Published 7 December 2020 \\ Copyright (C) 2020 Haoying Chen et al. This is an open access article distributed under the Creative Commons Attribution License, \\ which permits unrestricted use, distribution, and reproduction in any medium, provided the original work is properly cited.
}

In the article titled "Study on Inlet and Engine Integrated Model with Normal Shock Position Feedback" [1], there was an error in the Acknowledgments section. The corrected section appears below:

\section{Acknowledgments}

This study is supported in part by the National Science and Technology Major Project under Grant 2017-V-0004-0054, Research on the Basic Problem of Intelligent Aero-engine under Grant 2017-JCJQ-ZD-047-21, National Natural Science Foundation of China under Grant 51906102, China Postdoctoral Science Foundation Funded Project under Grant 2019M661835, and Aeronautics Power Foundation under Grant 6141B09050385.

\section{References}

[1] H. Chen, H. Zhang, Z. Xi, and Q. Zheng, "Study on inlet and engine integrated model with normal shock position feedback," International Journal of Aerospace Engineering, vol. 2020, Article ID 5313941, 13 pages, 2020. 